\title{
L-Acetyl-carnitine in Patients with Carpal Tunnel Syndrome: Effects on Nerve Protection, Hand Function and Pain
}

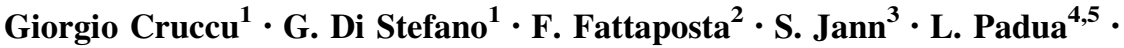 \\ A. Schenone ${ }^{6} \cdot$ A. Truini ${ }^{1}$
}

Published online: 20 December 2017

(c) The Author(s) 2017. This article is an open access publication

\begin{abstract}
Background and Aim L-Acetyl-carnitine (LAC) exerts an energetic effect on nerves and muscles. Recently, preclinical experiments have demonstrated a central anti-nociceptive action.

Objective Our objective was to assess the effects of LAC on neuroprotection, pain, and function in carpal tunnel syndrome (CTS), a very frequent chronic compressive neuropathy.

Methods In a multicentre, examiner-blinded, clinical and neurophysiological 4-month study, we enrolled 82 patients and examined 120 hands with CTS of mild to moderate severity. Patients were assessed at baseline and 10,60 and 120 days after treatment with LAC $500 \mathrm{mg}$ twice daily (BID). All patients underwent a conduction study of the median nerve, the Boston Carpal Tunnel Questionnaire
\end{abstract}

Electronic supplementary material The online version of this article (https://doi.org/10.1007/s40263-017-0476-2) contains supplementary material, which is available to authorized users.

Giorgio Cruccu

giorgio.cruccu@uniroma1.it

1 Department of Neurology and Psychiatry, Sapienza University, Viale Università 30, 00185 Roma, Italy

2 Policlinico Umberto I, Sapienza University, Roma, Italy

3 Department of Neuroscience, Niguarda Hospital, Milano, Italy

4 Department of Geriatrics, Neurosciences and Orthopaedics, Università Cattolica del Sacro Cuore, Rome, Italy

5 Department of Neurorehabilitation, Don Carlo Gnocchi Onlus Foundation, Milan, Italy

6 Department of Neuroscience, Rehabilitation, Ophthalmology, Genetics, Maternal and Child Health (DiNOGMI), University of Genova, Genova, Italy
(BCTQ) and the Neuropathic Pain Symptom Inventory (NPSI). The primary endpoint was the sensory conduction velocity (SCV) of the median nerve.

Results The primary endpoint was met, with significant improvement of the SCV $(P<0.0001)$. All sensory neurophysiological measures also significantly improved. BCTQ score changed significantly $(P<0.0001)$, with a greater improvement in the symptom component. Nine of the NPSI types of pain, particularly squeezing and pressure pain and pain evoked by pressure, showed a significant reduction $(P<0.0001)$.

Conclusions Our clinical and neurophysiological study indicated that 4 months of treatment with LAC exerted a neuroprotective effect. LAC reduced pain in patients with mild and moderate CTS, a result that is possibly due to both its neuroprotective action and its central anti-nociceptive properties.

Clinical Trials Registration code: EudraCT 2014-00228962.

\section{Key Points}

L-Acetyl-carnitine exerts a neuroprotective effect in patients with carpal tunnel syndrome of mild to moderate severity.

L-Acetyl-carnitine reduces pain in patients with mild and moderate carpal tunnel syndrome, possibly due to both its neuroprotective action and its central antinociceptive properties. 


\section{Introduction}

Compressive neuropathies have comparatively been neglected by drug companies, in terms of both neuroprotection and pain, whereas very many clinical trials have been performed in distal symmetric polyneuropathies, such as diabetic or chemotherapy-induced neuropathy. Estimates of the prevalence of diabetic neuropathy, the most frequent length-dependent neuropathy, range widely between 1.3 and 2\% [1-3]. Those for carpal tunnel syndrome (CTS), the most frequent compressive neuropathy, also range widely between 2 and 5\% [4-6], with a higher prevalence when diagnosed using a nerve conduction study (NCS) versus clinical examination [4]. Such values refer to the general population; specific working categories reach greater prevalence [7].

In neurological departments, these two neuropathies are, by far, the two most frequently diagnosed. Nevertheless, when we searched PubMed, only 19 clinical trials investigated chronic oral treatments in CTS compared with about 200 for diabetic neuropathy [8].

L-Acetyl-carnitine (LAC) has been found to exert a neuroprotective effect in patients with various types of peripheral neuropathy, including CTS [9]. Recently, studies in small mammals have demonstrated LAC's central action in experimental pain models, both neuropathic and inflammatory [10].

These two issues, together with the scarcity of trials in CTS, led us to test the efficacy of LAC on nerve conduction, pain and hand function in patients with mild to moderate CTS. The primary outcome was an improvement of the sensory conduction velocity (SCV) of the median nerve.

\section{Methods}

\subsection{Participants}

Sample size calculation led to an estimation of 70 patients plus $10-15 \%$ to compensate for probable discontinuations. Patients aged between 18 and 65 years, with a diagnosis of mild to moderate CTS as defined by the criteria of Padua et al. [11] and duration of symptoms no longer than 12 months were included in the study. Key exclusion criteria included other peripheral nerve diseases, medical conditions potentially associated with polyneuropathy and orthopaedic conditions affecting the wrist. Electronic Supplementary Material (ESM) 1 provides the list of all inclusion and exclusion criteria and the rules for concomitant drugs. Written informed consent was obtained from all participants before study-related procedures. The study protocol, patient information and informed consent forms were reviewed and approved by the Institutional Review Board at the participant hospitals.

\subsection{Study Design}

In this multicentre, examiner-blinded, clinical and neurophysiological study, eligible subjects were assessed at baseline and 10, 60 and 120 days after treatment (Fig. 1).

The study lasted 4 months, corresponding to the duration of the treatment. The screening period did not have a fixed duration, but in most cases the enrolment occurred during the screening visit. The recruitment period lasted 18 months, from May 2015 to November 2016.

The clinical trial included five neurology hospital clinics with expert staff for electrophysiology and pain.

The treatment phases consisted of an initial 10-day period of intramuscular injections of LAC $500 \mathrm{mg}$ twice daily (BID) followed by a 110-day period of oral treatment with one tablet of LAC $500 \mathrm{mg}$ BID. Patients who did not reach $80 \%$ of the target dosage at any visit were discontinued for poor compliance.

In total, 85 patients (125 hands) with mild to moderate CTS were screened. Three did not complete the procedures foreseen in the enrolment visit, resulting in screening failures. Therefore, 82 patients ( 25 men, 57 women; mean age $47.1 \pm 9.0$ years) were enrolled (corresponding to 120 hands, 63 mild CTS and 57 moderate CTS). Nine discontinued the treatment: eight withdrew consent and one was discontinued for poor compliance.

All patients underwent neurophysiological recording of sensory and motor nerve conduction as well as the Douleur Neuropathique 4 (DN4) to identify neuropathic pain [12], Neuropathic Pain Symptom Inventory (NPSI) to rate various types of pain [13] and Boston Carpal Tunnel Questionnaire (BCTQ) to assess hand function [14] at baseline and after 10,60 and 120 days. The three questionnaires can be found in ESM 2.

Safety evaluations, including assessments of adverse events, vital signs, laboratory measures, and physical examination findings were performed during each scheduled visit.

Possible adverse events, either volunteered spontaneously by patients or in response to general non-leading questioning by the investigator, were recorded from the start of treatment on day 0 until the final day-120 visit.

\subsection{Assessments}

\subsubsection{Neurophysiological Assessment}

At screening, patients underwent an NCS to both classify the severity of CTS and to control for other peripheral 
Fig. 1 Flow-chart of participants and study design. $B I D$ twice daily, ITT intention to treat, $N C S$ nerve conduction study, $t 0, t 10, t 60$, and $t 120$ indicate the day of visit

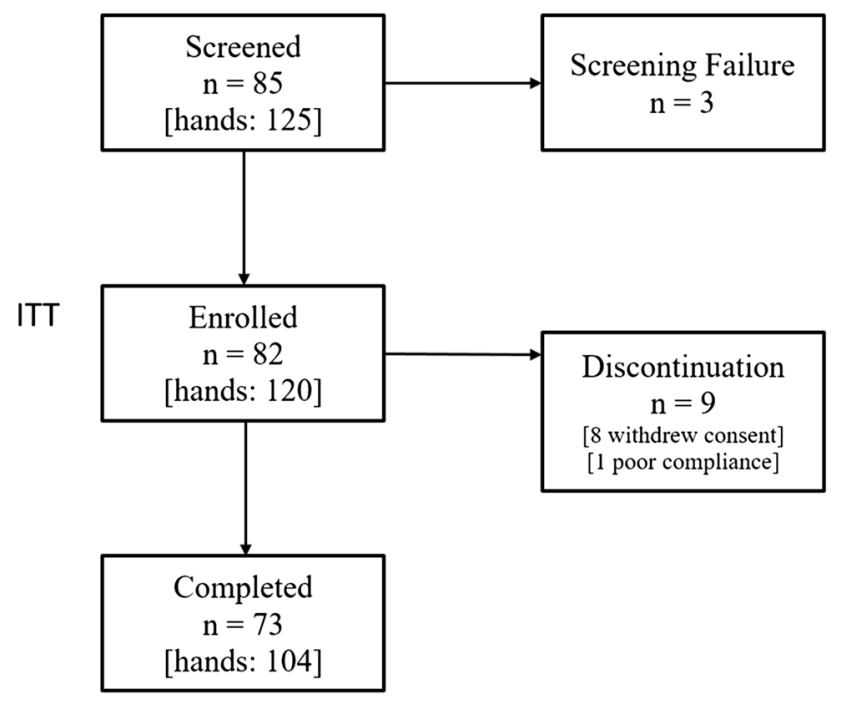

\begin{tabular}{|c|c|c|c|c|}
\hline & L-Ace & itine & & \\
\hline & $500 \mathrm{mg}$ & & al tablets BID & \\
\hline Screening & to & $\mathrm{t} 10$ & t60 & $\mathrm{t} 120$ \\
\hline & $\begin{array}{l}\text { onnaires } \\
\text { ng }\end{array}$ & $\begin{array}{l}\text { Visit } \\
\text { Questionnaires }\end{array}$ & $\begin{array}{l}\text { Visit } \\
\text { Questionnaires } \\
\text { NCS }\end{array}$ & $\begin{array}{l}\text { Visit } \\
\text { Questionnaires } \\
\text { NCS } \\
\text { Blood } \\
\text { sampling }\end{array}$ \\
\hline
\end{tabular}

neuropathies. Neurophysiological recordings and measurements were performed by specific medical staff. In particular, measures were taken by examiners who were blinded to the patient's condition. To assess CTS, patients underwent a motor and sensory NCS using surfacerecording electrodes with standard placement. NCS included amplitude and conduction velocity of sensory nerve action potentials (SAPs) recorded from the median nerve at the wrist, after first and third finger stimulation. We also measured the median-nerve compound motor action potential (CMAP) amplitude and latency after stimulation of the wrist and recording from thenar eminence. These methods adhered to those recommended by experts of the International Federation of Clinical Neurophysiology [15].

\subsubsection{Hand Dysfunction Assessment}

Hand dysfunction severity was evaluated during each scheduled visit using the BCTQ, the most commonly used questionnaire for the measurement of symptom severity and functional status with reproducibility, internal consistency, and validity in patients with CTS [14]. It includes two parts: symptom and functional evaluation. The first part comprises 11 items that can be scored from 1 (no symptom) to 5 (severe), with the total score ranging from
11 (best) to 55 (worst). The second part comprises eight items evaluating difficulty while performing daily tasks. The responses are also scored using a 5-point scale, for a total score ranging from 8 (best) to 40 (worst).

\subsubsection{Pain Assessment}

We used the DN4 questionnaire to identify neuropathic pain. DN4 is a clinician-administered questionnaire consisting of ten items: seven, concerning the quality of pain, are obtained by interviewing the patient, and three items are based on clinical examination and analyse the presence or absence of touch or pinprick hypoesthesia and tactile allodynia. A score of 1 is given to each positive item and a score of 0 to each negative item. Scores $\geq 4 / 10$ are considered indicative of neuropathic pain [12].

Pain intensity was rated at each scheduled visit by means of the NPSI questionnaire, a self-administered questionnaire specifically designed to evaluate the various types of pain [13]. This questionnaire includes a list of ten descriptors (plus two temporal items, questions 4 and 7) reflecting spontaneous ongoing or paroxysmal pain, evoked pain (i.e. mechanical and thermal allodynia/hyperalgesia) and dysesthesia/paresthesia. Each of these items is 
quantified on a numerical scale ranging from 0 to 10 , allowing discrimination and quantification of distinct pain qualities.

\subsection{Outcomes}

The SCV of the median nerve was the primary endpoint. SAP amplitude and CMAP latency and amplitude were assessed as secondary endpoints. The secondary outcomes also included the BCTQ to monitor symptom severity and the NPSI as a measure of intensity of the various types of pain. Outcome variables were monitored at baseline, 10, 60 and 120 days (Fig. 1). We also verified whether the severity of CTS (mild vs. moderate) had an impact on the outcome measures.

\subsection{Statistics}

All neurophysiological measures, being linear and normally distributed, were evaluated with analysis of variance (ANOVA) for repeated measures with post hoc analysis for linear trend. All the questionnaire scores, being non-linear and occasionally non-normally distributed, were evaluated with the Friedman test, Dunn's multiple comparison test and Wilcoxon's matched pairs test. The differences between mild and moderate CTS were evaluated with the Mann-Whitney $U$ test.

The missing values of the nine patients who discontinued were treated as last observation carried forward (LOCF), usual preference for linear measures provided by laboratory tools such as those for neurophysiological measures.

All statistics and graphs were created using Prism (GraphPad, Sorrento Valley, CA, USA).

\section{Results}

\subsection{Primary Outcome}

In the 82 enrolled patients, the SCV of the median nerve, as measured after stimulation of the third finger, was $36.6 \pm 5.2 \mathrm{~m} / \mathrm{s}$ at baseline (t0) and increased to $38.9 \pm 6.8 \mathrm{~m} / \mathrm{s}$ after 4 months of treatment (t120). As evaluated with ANOVA for repeated measures, the difference was highly significant $(P<0.0001)$. After stimulation of the first finger, the SCV changed from $34.7 \pm 5.6 \mathrm{~m} / \mathrm{s}$ at to to $36.7 \pm 6.6 \mathrm{~m} / \mathrm{s}$ at $\mathrm{t} 120(P<0.0001)$. The values were distributed linearly along time (Fig. 2), with a positive linear trend to increase $(P<0.0001)$ for both fingers.

\subsection{Other Neurophysiological Measures}

From baseline to the end of the study, the amplitude of sensory action potentials changed from $11.8 \pm 8.6$ to $14.1 \pm 9.3 \mu \mathrm{V}(P<0.0001)$ after stimulation of the third finger and from $11.2 \pm 6.2$ to $13.7 \pm 8.7 \mu \mathrm{V}(P<0.0001)$ after stimulation of the first finger, with a positive linear trend to increase for both fingers.

The CMAPs did not improve as much as the sensory. The latency shortened from $4.1 \pm 0.9$ to $3.9 \pm 0.8 \mathrm{~ms}$ $(P<0.02)$. The amplitude change, from $8.2 \pm 3.5$ to $8.7 \pm 3.3 \mathrm{mV}$, was not statistically significant.

\subsection{Hand Dysfunction}

Both the symptom and functional BCTQ scores significantly decreased. The symptom BCTQ score decreased by $39 \%$, from 19.3 (95\% CI 17.6-21.1) at to to 11.8 (95\% CI 10.3-13.2) at $\mathrm{t} 120(P<0.0001)$. The functional BCTQ score decreased by $18 \%$, from $15.2(95 \%$ CI $13.9-16.5)$ at t0 to $12.5(95 \%$ CI $11.5-13.5)$ at $1120(18 \% ; P<0.0001)$.

The Dunn's multiple comparison test showed that the time course of symptom and functional BCTQ scores did not decrease between t60 and t120 (Fig. 3).

\subsection{Pain}

Of the 82 enrolled patients, only 44 (54\%), corresponding to 53 hands, had neuropathic pain, as assessed with DN4.

In all patients, we used the NPSI to assess the various types of pain. We included in the NPSI analysis only the hands scoring at least 4 in any 0-10 scale of the ten types of pain considered in the NPSI. Therefore, our population assessed with the NPSI included 77 hands in 58 patients. At baseline, the type of pain scoring highest was tingling (6.8), followed by pain evoked by pressure (4.1) and pins and needles (3.7). Notably, burning pain, which is probably the most frequent type of neuropathic pain, scored only 2.9. Stabbing pain scored least (1.7) (Table 1).

In the course of the 4-month treatment period, all types of pain decreased significantly, with the exception of stabbing pain (Table 1). The effect size ranged from 38 to $56 \%$, with the greatest pain relief reported for spontaneous squeezing and pressure pains and pain evoked by pressure (Table 1).

Estimated by the Wilcoxon test, the paired difference between $\mathrm{t} 0$ and $\mathrm{t} 120$ were always significant, except for stabbing pain (Table 1). 
Fig. 2 Results of the mediannerve conduction study in 82 patients. ANOVA analysis of variance, $C M A P$ compound motor action potential, $S A P$ amplitude of the sensory action potentials, $S C V$ sensory conduction velocity. Squares are mean \pm standard error. $P$ ANOVA for repeated measures. Note that all sensory measures improve significantly and linearly, whereas the changes in amplitude of motor potentials are not significant
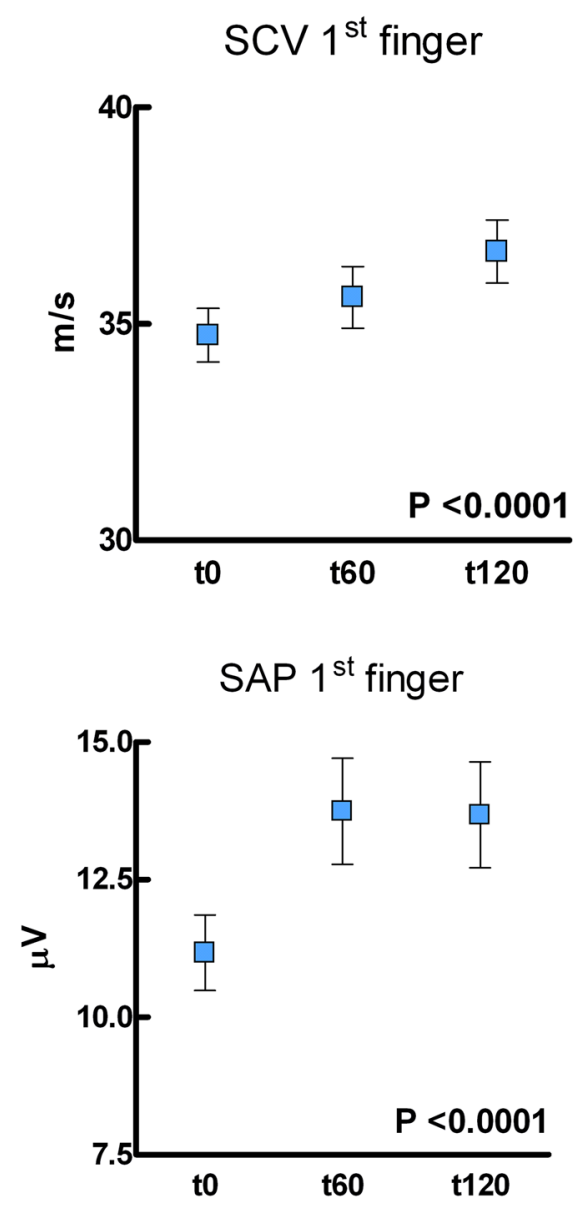

CMAP Latency

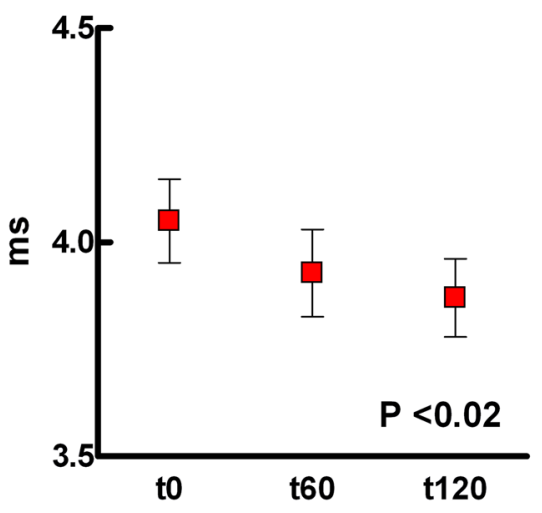

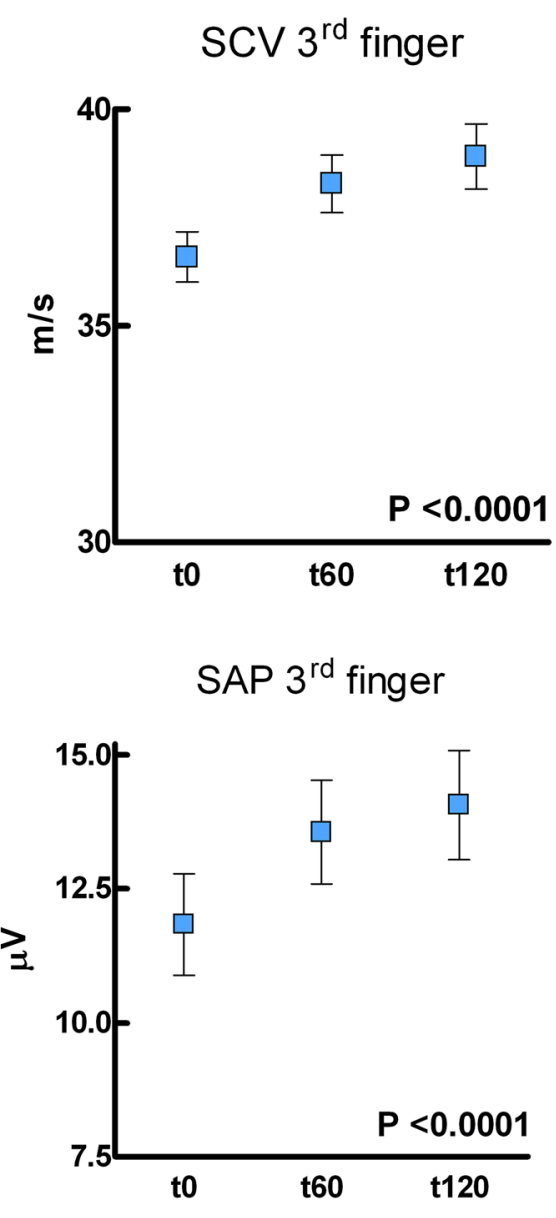

CMAP Amplitude

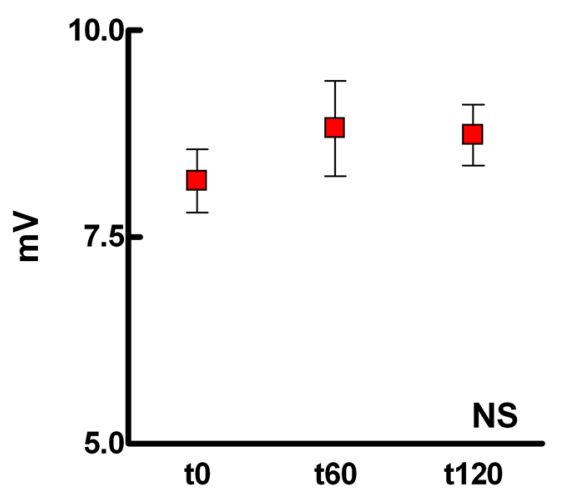

\subsection{Comparison Between Mild and Moderate Carpal Tunnel Syndrome (CTS)}

The severity of CTS did not affect the neurophysiological or subjective outcome measures, except for a greater shortening of CMAP latency in the hands with moderate CTS $(P=0.026)$.

\subsection{Adverse Events}

No serious adverse events were reported during this study. In total, $15(18.3 \%)$ of the 82 patients enrolled reported a total of 19 adverse events (18 were classified as mild and one as moderate). None of these adverse events was considered related to treatment. Urinary tract infection was the most common adverse event, occurring in eight $(9.8 \%)$ patients, followed by an increase of total bilirubin plasma 
Fig. 3 Boston Carpal Tunnel Questionnaire (BCTQ). Squares are mean $\pm 95 \%$ confidence intervals. $P$ Friedman test. Note that the effect size, although statistically significant for both BCTQ parts, is greater for the symptom than for the functional part
Symptom BCTQ

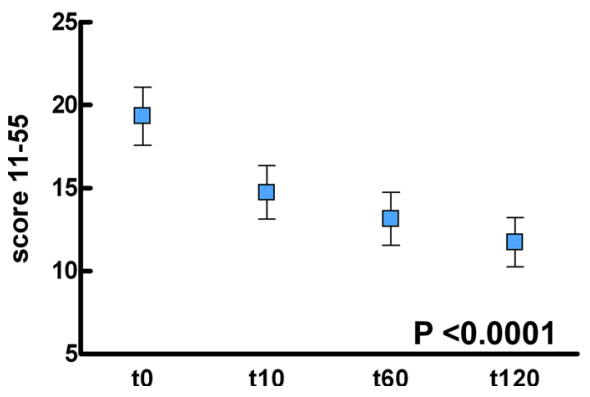

Functional BCTQ

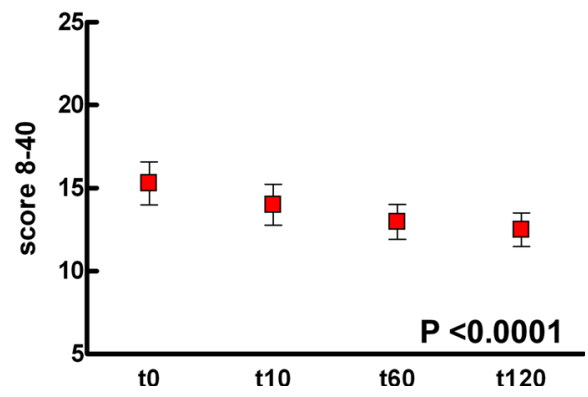

level in three (3.7\%) and hypercholesterolemia in another three $(3.7 \%)$ patients. Remaining adverse events included single cases of leukopenia, increased transaminases, hyperglycaemia, colitis and haematuria. These adverse events were evidenced by abnormal laboratory findings. All patients were asymptomatic (including those with urinary infections) (ESM 3).

\section{Discussion}

In this multicentre, examiner-blinded, clinical and neurophysiological study, we showed that 120 days of treatment with LAC significantly improved the median-nerve SCV in patients with mild or moderate CTS. LAC treatment also had a positive impact on the patient-oriented measures related to sensory function and pain.

\subsection{Objective and Subjective Measures}

Our clinical and neurophysiological study is an uncontrolled trial with no control group for comparison. We admit that, in CTS, where sensory symptoms play a major role, this is a serious limitation. However, bearing this limitation in mind, we identified the SCV of the median nerve as the primary endpoint and reported outcomes from all patients as secondary outcome measures.

The SCV is an objective measure, uninfluenced by possible placebo effects. Furthermore, the investigators recording the NCS were not the same as those measuring the NCS variables, and all were blinded to the patient's condition. Conversely, outcomes reported by patients may include placebo effects. Accordingly, to address this problem, we used the BCTQ to compare symptoms and function and the NPSI questionnaire to compare the various types of pain and verify whether the 4-month treatment distinctly influenced the different variables. While we admit that further, larger and controlled studies are needed to confirm the efficacy of LAC on pain, we believe that our data reliably support a neuroprotective effect of LAC.
The evidence of a neuroprotective effect of LAC relies on the NCS changes. CTS-related variables (e.g. NCS) are expected to remain unchanged over a short period and worsen over a longer period [16-18]. In contrast, the NCS variables in our patients significantly improved after treatment.

\subsection{Neuroprotective Effects}

The linear improvement of sensory NCS variables with no tendency to plateauing during the 4-month treatment period support the neuroprotective effect of LAC. This effect is probably mediated through various mechanisms that ultimately restore mitochondrial activity [19, 20]. However, our NCS data cannot show whether LAC treatment promotes axonal regeneration or remyelination. Alternatively, we cannot exclude that nerve fibers might have simply benefitted from removal of conduction blocks.

In our study, we used median nerve SCV as the main outcome measure. Admittedly, the relationship between this neurophysiological variable and patients' reported symptoms (pain included) remains controversial [21]; however, the nerve conduction velocity provides an objective measure, which is also widely used in animal studies [22].

Among the different NCS variables, distal motor latency showed a small but significant improvement during the treatment period. Conversely the amplitude of the compound muscle potentials did not significantly change (Fig. 2). Our patients, who had mild or moderate CTS, may have an increased latency of CMAPs; however, they were required to have normal amplitude of CMAPs at the time of inclusion (ESM 1).

Although distal motor latency change demonstrates that LAC also exerts a positive effect on motor nerve fibers, the effect size is smaller than that on sensory nerve fibers.

\subsection{Pain in CTS}

Pain did not seem to be the main problem for the majority of our patients with mild to moderate CTS. Almost half of 


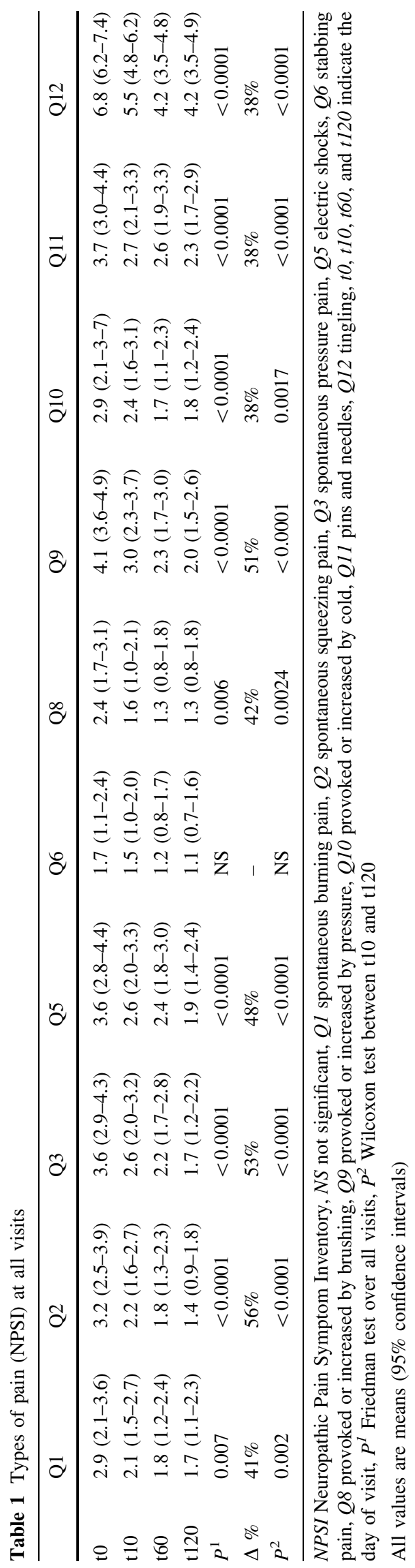

the patients did not reach the DN4 threshold for neuropathic pain, and an important proportion of hands and patients did not reach a score of 4 in any of the ten types of pain investigated with the NPSI. Patients with CTS may experience both neuropathic and non-neuropathic (inflammatory or nociceptive) pain [6]. Given that previous studies have also used NPSI for predominantly nociceptive pain [23], we also applied this questionnaire to hands that scored below the DN4 threshold. However, in the NPSI analysis, we included only hands scoring at least 4 on any of the NPSI measures of pain intensity, thus adhering to the traditional cutoff adopted in clinical trials for pain.

At baseline, NPSI analysis showed that the most frequent and severe type of sensory disturbance was tingling, supposedly mediated by $A \beta$ fibers [24]. We hypothesize that this finding might reflect the median nerve compression, which is expected to damage progressively from the largest- to the smallest-diameter nerve fibers [25].

LAC treatment predominantly relieved pressure and squeezing pain and pain evoked by pressure. However, it is difficult to correctly interpret the origin of these types of pain. CTS commonly causes neuropathic pain due to median nerve damage and nociceptive pain due to inflammatory changes of the carpal tunnel; the NPSI refers to the "area of pain" rather than the projected innervation territory of the median nerve, and patients described their pain in the whole hand, including the wrist. Therefore, we hypothesize that these types of pain might have, in our patients, a nociceptive origin. Accordingly, we conjecture that question 9 of the NPSI (is your pain provoked or increased by pressure on the painful area?), scored high probably because patients felt more pain when pressing on their wrist. The concomitant low scores for brush and cold allodynia (questions 8 and 10) indirectly support this view.

Admittedly, NPSI findings based on patients' perception of pain do not provide reliable evidence on the origin of pain, whether nociceptive or neuropathic pain. However, we believe that LAC-induced changes of these NPSI items support the anti-nociceptive activity of this drug [10].

The anti-nociceptive activity of LAC might result from different mechanisms. LAC selectively induces the expression of mGlu2 by enhancing the activity of the nuclear factor (NF)- $\kappa \mathrm{B}$ family of transcription factors. mGlu2 receptors localized in the spinal cord and other regions of the nociceptive system negatively regulate glutamate release [10]. However, other animal studies showing that the LAC effect on pain is modulated by nicotinic and muscarinic antagonists indicate that the antinociceptive activity of this drug might be mediated through the cholinergic pathway [26, 27]. 


\section{Conclusions}

A virtually harmless treatment period (4 months) with LAC in patients with mild to moderate CTS significantly improved neurophysiological measures, thus demonstrating a neuroprotective action. We also found a positive impact on the patient-oriented measures related to sensory function and pain. Although, in clinical practice, most clinicians are accustomed to think of CTS as a condition that entails surgery, pharmacological treatments should be taken into account.

Acknowledgements Collaborators: Nicoletta Locuratolo, Gabriele Valente (Policlinico Umberto I, Sapienza University, Roma, Italy); Valeria Mariani (Department of Neuroscience, Niguarda Hospital, Milano, Italy); Giulia Ursino, Lizia Reni, Michela Ferrara (Department of Neuroscience, Rehabilitation, Ophthalmology, Genetics, Maternal and Child Health, University of Genova, Genova, Italy).

\section{Compliance with Ethical Standards}

Funding The trial was funded by Sigma-Tau of ALFASIGMA Group. Open access was funded by Sigma-Tau of ALFASIGMA Group.

Conflict of interest Giorgio Cruccu has received a research grant, consulting fees and payments for lectures from Sigma-Tau of ALFASIGMA Group, and consulting fees from Angelini, Biogen, and Mundipharma. Giulia Di Stefano, Francesco Fattapposta, and Luca Padua have no conflicts to declare. Stefano Jann has received payments for lectures and consulting fees from Sigma-Tau of ALFASIGMA Group and Gruenenthal and a research grant from Grifols. Angelo Schenone has received payments for lectures from Sigma-Tau of ALFASIGMA Group, Mundipharma, Ecupharma, Kedrion, CSL Behering, and Epitech. Andrea Truini has received payments for lectures from Sigma-Tau of ALFASIGMA Group and consulting fees or payment for lectures from Angelini, Gruenenthal and Pfizer.

Ethics approval The study protocol, patient information and informed consent forms were reviewed and approved by the Institutional Review Board at the participant hospitals.

Consent to participate All participants provided written informed consent before taking part in study procedures. All procedures were in accordance with Good Clinical Practice and the principles of the Declaration of Helsinki.

Open Access This article is distributed under the terms of the Creative Commons Attribution-NonCommercial 4.0 International License (http://creativecommons.org/licenses/by-nc/4.0/), which permits any noncommercial use, distribution, and reproduction in any medium, provided you give appropriate credit to the original author(s) and the source, provide a link to the Creative Commons license, and indicate if changes were made.

\section{References}

1. MacDonald BK, Cockerell OC, Sander JW, Shorvon SD. The incidence and lifetime prevalence of neurological disorders in a prospective community-based study in the UK. Brain. 2000;123:665-76.

2. Davies M, Brophy S, Williams R, Taylor A. The prevalence, severity, and impact of painful diabetic peripheral neuropathy in type 2 diabetes. Diabetes Care. 2006;29:1518-22.

3. Sadosky A, McDermott AM, Brandenburg NA, Strauss M. A review of the epidemiology of painful diabetic peripheral neuropathy, postherpetic neuralgia, and less commonly studied neuropathic pain conditions. Pain Pract. 2008;8:45-56.

4. Atroshi I, Gummesson C, Johnsson R, Ornstein E, Ranstam J, Rosén I. Prevalence of carpal tunnel syndrome in a general population. JAMA. 1999;282:153-8.

5. Bland JDP, Rudolfer SM. Clinical surveillance of carpal tunnel syndrome in two areas of the United Kingdom, 1991-2001. J Neurol Neurosurg Psychiatry. 2003;74:1674-9.

6. Padua L, Coraci D, Erra C, Pazzaglia C, Paolasso I, Loreti C, et al. Carpal tunnel syndrome: clinical features, diagnosis, and management. Lancet Neurol. 2016;15:1273-84.

7. Hagberg M, Morgenstern H, Kelsh M. Impact of occupations and job tasks on the prevalence of carpal tunnel syndrome. Scand $\mathrm{J}$ Work Environ Health. 1992;18:337-45.

8. Truini A, Barbanti P, Pozzilli C, Cruccu G. A mechanism-based classification of pain in multiple sclerosis. J Neurol. 2013;260:351-67.

9. De Grandis D, Santoro L, Di Benedetto P. L-acetylcarnitine in the treatment of patients with peripheral neuropathies : a short term, double-blind clinical study of 426 patients. Clin Drug Investig. 1995;10:317-22.

10. Notartomaso S, Mascio G, Bernabucci M, Zappulla C, Scarselli $\mathrm{P}$, Cannella M, et al. Analgesia induced by the epigenetic drug, Lacetylcarnitine, outlasts the end of treatment in mouse models of chronic inflammatory and neuropathic pain. Mol Pain. 2017;13:1744806917697009. https://doi.org/10.1177/ 1744806917697009 .

11. Padua L, Monaco ML, Padua R, Gregori B, Tonali P. Neurophysiological classification of carpal tunnel syndrome: assessment of 600 symptomatic hands. Ital J Neurol Sci. 1997; 18:145-50.

12. Bouhassira D, Attal N, Alchaar H, Boureau F, Brochet B, Bruxelle $\mathrm{J}$, et al. Comparison of pain syndromes associated with nervous or somatic lesions and development of a new neuropathic pain diagnostic questionnaire (DN4). Pain. 2005;114:29-36.

13. Bouhassira D, Attal N, Fermanian J, Alchaar H, Gautron M, Masquelier E, et al. Development and validation of the neuropathic pain symptom inventory. Pain. 2004;108:248-57.

14. Levine DW, Simmons BP, Koris MJ, Daltroy LH, Hohl GG, Fossel AH, et al. A self-administered questionnaire for the assessment of severity of symptoms and functional status in carpal tunnel syndrome. J Bone Jt Surg Am. 1993;75:1585-92.

15. Kimura J, editor. Peripheral nerve diseases, handbook of clinical neurophysiology. Amsterdam: Elsevier; 2006.

16. Padua L, Padua R, Aprile I, Tonali P. Italian multicentre study of carpal tunnel syndrome: differences in the clinical and neurophysiological features between male and female patients. J Hand Surg Br. 1999;24:579-82.

17. Padua L, Padua R, Aprile I, Pasqualetti P, Tonali P, Italian CTS Study Group. Carpal tunnel syndrome. Multiperspective followup of untreated carpal tunnel syndrome: a multicenter study. Neurology. 2001;12(56):1459-66.

18. Padua L, Padua R, Monaco ML, Aprile I, Paciello N, Nazzaro M, et al. Natural history of carpal tunnel syndrome according to the neurophysiological classification. Ital $J$ Neurol Sci. 1998;19:357-61.

19. Patel SP, Sullivan PG, Lyttle TS, Magnuson DS, Rabchevsky AG. Acetyl-L-carnitine treatment following spinal cord injury improves mitochondrial function correlated with remarkable 
tissue sparing and functional recovery. Neuroscience. 2012;210:296-307.

20. Di Cesare Mannelli L, Ghelardini C, Calvani M, Nicolai R, Mosconi L, Vivoli E, et al. Protective effect of acetyl-L-carnitine on the apoptotic pathway of peripheral neuropathy. Eur J Neurosci. 2007;26:820-7.

21. Truini A, Garcia-Larrea L, Cruccu G. Reappraising neuropathic pain in humans-how symptoms help disclose mechanisms. Nat Rev Neurol. 2013;9:572-82.

22. Brussee V, Guo G, Dong Y, Cheng C, Martinez JA, Smith D, et al. Distal degenerative sensory neuropathy in a long-term type 2 diabetes rat model. Diabetes. 2008;57:1664-73.

23. Rüger LJ, Irnich D, Abahji TN, Crispin A, Hoffmann U, Lang PM. Characteristics of chronic ischemic pain in patients with peripheral arterial disease. Pain. 2008;30(139):201-8.
24. Truini A, Padua L, Biasiotta A, Caliandro P, Pazzaglia C, Galeotti F, et al. Differential involvement of A-delta and A-beta fibres in neuropathic pain related to carpal tunnel syndrome. Pain 2009;145(1-2):105-9.

25. Ochoa J, Fowler TJ, Gilliatt RW. Anatomical changes in peripheral nerves compressed by a pneumatic tourniquet. J Anat. 1972;113:433-55.

26. Di Cesare Mannelli L, Ghelardini C, Calvani M, Nicolai R, Mosconi L, Toscano A, et al. Neuroprotective effects of acetyl-Lcarnitine on neuropathic pain and apoptosis: a role for the nicotinic receptor. J Neurosci Res. 2009;87:200-7.

27. Bartolini A, Di Cesare Mannelli L, Ghelardini C. Analgesic and antineuropathic drugs acting through central cholinergic mechanisms. Recent Pat CNS Drug Discov. 2011;6:119-40. 